\title{
OPERATIVE CHOLANGIOGRAPHY
}

\author{
By A. W. NuRICK, F.R.C.S. \\ Late Senior Registrar, The Middlesex Hospital
}

There is no doubt that stones may be left behind in the common bile duct after operation. Estimates of the incidence of this complication vary widely and the following table shows some of the published figures:

Incidence of Residual Stone in the Common Duct

\begin{tabular}{|c|c|}
\hline Authority & Incidence \\
\hline 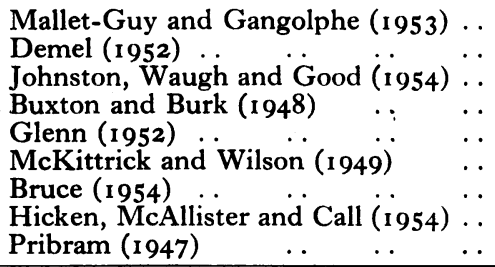 & $\begin{array}{l}6.9 \% \\
5.5 \% \\
8 \% \\
5.9 \% \\
7 \% \\
2 \% \\
2.5 \% \\
20 \% \\
16-25 \%\end{array}$ \\
\hline
\end{tabular}

It is difficult to interpret the higher figures except as a reflection on the frequency and thoroughness of duct exploration or on the experience of the surgeon. Hicken, McAllister and Call (1954) found that in their collected series of $55^{\circ}$ operations with residual stones in 110 ( 20 per cent. incidence) the chance of leaving a stone increased tenfold when the surgeon was inexperienced. The overall incidence of residual stone is probably in the neighbourhood of 5 per cent.; lower in the hands of experts, higher in the hands of those less accustomed to biliary surgery.

The possible causes of residual stones in the common bile duct are two: failure to explore the duct and failure, on exploration, to remove all stones. The third possibility of stone formation in the common duct after cholecystectomy does exist, but the majority of stones found in the common duct after cholecystectomy have been there since the original operation.

It is not within the scope of this paper to examine the indications for exploration of the common bile duct, for they are widely known (Allen, I936; Lahey, I938; Glenn, I952). If these indications are recognized and conscientiously observed, few stones should be left behind as a result of failure to open the duct; by the same token a number of fruitless explorations are bound to be performed.
Exploration of the common bile duct does not $\stackrel{2}{\%}$ necessarily mean that it has been completely $\vec{O}$ cleared of stones and the practice of T tube drain- $\overrightarrow{\vec{H}}$ age of the duct has been generally adopted because ${ }_{\sigma}$ of the danger of intraperitoneal leakage of bile $\vec{B}$ from the suture line should the duct be closed and 3 obstruction to the flow of bile into the duodenum develop.

It is customary, before removing a $T$ tube, to $\stackrel{\partial}{\partial}$ perform cholangiography. This investigation is $\omega_{0}$ usually responsible for making the diagnosis of a $\infty$ stone in the common duct which has been missed 을 at operation. Post-operative cholangiography is performed about 10 days after operation and when it demonstrates a residual stone the surgeon is ${ }_{C}$ presented with information which would have been $\stackrel{5}{\rightarrow}$ both more useful and more welcome had it beeg $\vec{c}$ available while the abdomen was still open at the f original operation.

Hicken, McAllister, Franz and Crowder (195 have traced the development of post-operative cholangiography since the accidental demonstration by Reich in 1918 of the biliary tract after the injection of 'petrolatum and barium paste' into a persistent sinus. Mirrizi (1932) seems to have been the first to take the logical step of performing cholangiography at the time of operation. The early operative cholangiograms often failed to outline the whole biliary tract because the medium used was lipiodol, which may pool and globulate in the ducts because it will not mix with bile. The introduction of $a^{\text {* }}$ water-soluble organic iodine compound, diodone, 35 per cent. or 50 per cent., has greatly improved the quality of cholangiograms (Hicken et al., 1950).

Although post-operative cholangiography is now $\frac{D}{2}$ a routine procedure, cholangiography during operation has not achieved much popularity out- $\widetilde{N}$ side a few centres. Its chief advocates have been $N$ Mallet-Guy (1947, 1952) in France, Hicken and N his co-workers (1949) in America, Milbourn (1949) and Norman (I95I) in Sweden, and McNeill Love (1952) in this country.

Cholangiography may be performed as a diag- $\stackrel{\mathbb{D}}{+}$ nostic measure before duct exploration, by means of a cannula or polythene tube inserted into the 
cystic duct, or by direct injection of diodone into the common duct. Diagnostic cholangiography causes little delay in the operation, for the surgeon can remove the gall bladder while the films are being developed. Post-exploratory or control cholangiography is performed via a $\mathrm{T}$ tube sutured firmly into the common duct so as to prevent any leakage; there is an inevitable delay in the operation while the films are developed.

The theoretical grounds for advocating cholangiography at the time of operation rather than Io days later are unquestionably sound, but it is apparent from some of the published work that the procedure is not absolutely reliable. "False positive' cholangiograms may be obtained which indicate a stone when none is present and 'false negative' cholangiograms may be obtained which suggest the duct is clear of stones when they are, in fact, present. Reviewing 406 cases, Mixter, Hermanson and Segel (195I) found that in II3 cases where operative cholangiography was followed by duct exploration there were five ' false positive' and nine 'false negative' cholangiograms, a total error of 12 per cent. in the procedure. Operative cholangiography after exploration of the duct in 146 cases revealed unsuspected residual calculi in 19 ( 13 per cent.), but there were also seven patients in this group (4.9 per cent.) with apparently normal post-exploratory cholangiograms who were subsequently proved to have residual stones in the common duct at operation or post-mortem. Kantor, Evans and Glenn (1955) reported ${ }_{5} 8$ cases with 9 per cent. residual stones despite operative cholangiography. Hughes (1955) had three false negative and four false positive cholangiograms in 9I cases, an error of 8 per cent. In a personal experience of 65 operative cholangiograms where the duct was explored there were nine false positive and one false negative cholangiograms, an error of 15 per cent.

It seems, therefore, that the chance of getting a misleading operative cholangiogram is as great, if not greater, than the chance of leaving a stone in the duct after exploration. Although it is true that unexpected stones in the duct may be detected and removed as a result of operative cholangiography, it is also true that misplaced faith in an apparently normal cholangiogram may result in a stone being left behind.

One explanation why operative cholangiography is less accurate in the diagnosis of common duct stones than post-operative cholangiography lies in the difference between the two techniques. Postoperative cholangiography is performed under fluoroscopic control. The radiologist is able to position the patient and fill the ducts so as to get the best possible pictures; he can also observe the flow of diodone into the duodenum and may be able to tell whether a filling defect is an air bubble or a stone by its behaviour when the patient's position is altered. It is not practicable to perform operative cholangiography under screen control and few hospitals have the necessary equipment; the alternative used is to expose a series of films after injection of increasing volumes of diodone.

Errors of technique can result in misleading cholangiograms, air bubbles may simulate stones, movement may occur during exposure of the films, the shadow of the duct may be superimposed on the vertebral column and diodone, which has entered the duodenum, may overlie and obscure the lower end of the common duct where stones are so frequently to be found. False negative cholangiograms can also occur because the density of the medium may completely hide stones, especially when the duct is dilated. This has been shown experimentally by Gius, Tidrick and Hickey (1954).

Operative cholangiograms may be difficult to interpret. The normal duct is not dilated, free from filling defects and a small injection of diodone enters the duodenum easily. Stones in the common duct may reveal their presence by any one or any combination of the following signs : a filling defect, failure or poor entry of diodone into the duodenum, a dilated duct, excessive filling of the smaller intrahepatic ducts, irregularity or abrupt truncation of the lower end of the duct.

Cholangiograms performed after duct exploration should theoretically give the most useful information to the surgeon, but these cholangiograms are often the most difficult to interpret. It may be that instrumentation of the lower end of the duct sets up some local spasm, for it is not uncommon to find that the diodone fails to enter the duodenum even though instruments can be passed through the sphincter. This effect may be seen even after the duodenum has been opened and the sphincter of Oddi divided so that there can be no question about the patency of the lower end of the common duct. It is also difficult to exclude air bubbles and to avoid leakage of diodone in postexploratory cholangiograms.

The incidence of false negative and false positive cholangiograms can be diminished by careful attention to details of technique. Air bubbles can be excluded by filling all tubing with saline and by aspirating before injecting the diodone. Tilting the operating table laterally through $15^{\circ}$ to the right will throw the shadow of the common duct clear of the vertebral column. Serial films exposed after injection of $\mathrm{I}-3 \mathrm{ml}$., $6-10 \mathrm{ml}$. and $\mathrm{I}_{5}-20 \mathrm{ml}$. help to show the lower end of the duct clearly. Control of the patient's respiration during exposure of the films is essential. By these means, 
technically satisfactory films can be obtained in a high proportion of cases.

It is essential that, in addition to having good $\mathrm{X}$-ray films, the surgeon be familiar with the radiological appearance, normal and abnormal, of the biliary tract. Even when these conditions are fulfilled, there remains a margin for error in the examination itself or in the interpretation of the films. The result of this error is that a normal diagnostic operative cholangiogram is not necessarily a guarantee that the duct is free from stones and consequently does not excuse the surgeon from exploring the duct if any of the clinical or operative indications for exploration are present. A normal control cholangiogram after exploration of the duct, likewise, does not necessarily guarantee that the duct is clear. A false positive cholangiogram is a nuisance, for it may lead to an unnecessary exploration of the common duct, but a false negative cholangiogram is a menace, for it may result in a second operation for a residual stone.

The unreliability of operative cholangiography has influenced some authorities against its use. Waugh, Walters, Gray and Priestley (1952) consider it unnecessary and Maingot (I 952) considers it cumbersome, unnecessary and misleading. These facts do not, however, mean that operative cholangiography is devoid of value. Nobody who has had the experience of demonstrating an unsuspected residual calculus in the common duct by operative cholangiography and of removing the stone before closing the abdomen can question the value of cholangiography on such occasions. Whether it reveals stones or not, cholangiography will always show the anatomy of the duct system and will demonstrate any variation from the normal (Hicken, Coray and Franz, 1949) far more quickly and certainly than dissection will. It must not be forgotten that cholangiography has played the major part in bringing to the attention of surgeons the ease and frequency with which stones may be left behind in the common duct.

The hope that operative cholangiography would reduce substantially the number of unnecessary duct explorations and eliminate the risk of residual stones has not been fulfilled. The successes of the procedure show that it has great potentialities, but small stones or even large stones in dilated ducts may not be demonstrated. In a recent editorial, Walters (1955) states that after careful instrumental exploration of the common duct (through an incision in its wall and not through the stump of the cystic duct), repeated lavage and dilatation of the sphincter of Oddi the only stones liable to be left behind are small ones which can pass through the dilated sphincter into the duodenum. This view is supported by the experience of Brush, Powka, Damazo and Whitcomb (1955), who do not use operative cholangiography. They found that no second operations for residual stone were required $\mathrm{Q}$ in 198 cases where the sphincter of Oddi was dilated, whereas in a control series of 250 explora $-\overrightarrow{\vec{n}}$ tions where the sphincter was not dilated thereo were Io second operations for residual calculio (4 per cent.).

\section{Conclusions}

I. The value of operative cholangiography in the diagnosis of residual stone in the common bile. duct is diminished by the occurrence of false $\vec{\omega}$ negative and false positive results.

2. The use of operative cholangiography does not necessarily lessen the incidence of residual 3 . common duct stone.

3. The results of careful instrumental explora- $\stackrel{N}{\omega}$ tion of the common bile duct, including lavage and dilatation of the sphincter of Oddi, can be excellent.

\section{BIBLIOGRAPHY}

ALLEN, A. W. (1936), Surg. Gynec. Obstet., 62, 347. BRUCE, J. (1954), Trans. Med. Soc. Lond., 71, 19. BRUSH, B. E., and POWKA, J. L.

DAMAZO, F., and WHITCOMB, J. (1955), Arch. Surg. (Chicagg),

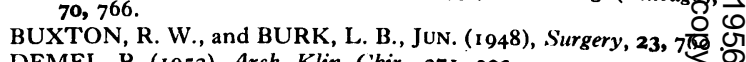
DEMEL, R. (1952), Arch. Klin. Chir., 271, 302. GIUS, T. A., TIDRICK, R. T., and HICKEY, R. C. (I9)
Surgery, 36, 460 .

GLENN, F. (1952), Surg. Gynec. Obstet., 95, 431. HICKEN, N. F., CORAY, Q. B., and FRANZ, B. (1949), Surg.
Gynec. Obstet., 88, 577.

HICKEN, N. F., MCALLISTER, A. J., FRANZ, B., and $\frac{\mathrm{\varrho}}{\mathrm{D}}$ CROWDER, E. (1950), Arch. Surg. (Chicago), 60, 1 102.

HICKEN, N. F., MCALLISTER, A. J., and CALL, W. D. (1954), 는 Ibid., 68, 643.

HUGHES, E. S. R. ('1955), Med. F. of Australia, I, 820.

KANTOR, H. G., EVANS, J. A., and GLENN, F. (1955), Arch. Surg. (Chicago), 70, 237.

LAHEY, F. H. (1938), Amer. F. Surg., 40, 209.

LOVE, R. J. MCNEILL (1952), Brit. F. Surg., 40, 214.

MAINGOT, R. (1952), Ann. R. Coll. Surg. Eng., ro, 97.

MALLET-GUY, P. (1947), "La Chirurgie biliaire sous controle Manometrique et Radiologique per operatoire,' Masson et Cie. Paris.

MALLET-GUY, P. (1952), Surg. Gynec. Obstet., 94, 385

MALLET-GUY, P., and GANGOLPHE, M. (1953), Mem. Acad. Chir., 79, 60.

MCKITTRICK, L. S., and WILSON, N. J. (I949), California Med., 71, 132

MILLBOURN, E. (1949), Acta. Chir. Scand., 99, 285.
MIRIZZI, P. L. (1932), Bol. Y. Trab. Soc. de Cir. de Buenos Aires, 号 I6, I 133 .

MIXTER, C. G., HERMANSON, L., and SEGEL, A. L. (I95 I), N
Ann. Surg., 134, 346. NORMAN, O. (1951), Acta. Radiol. Suppl., 84.

PRIBRAM, B. O. C. (1947), Surgery, 22, 806.

REICH, A. (1918), F.A.M.A., 71, 1555 .

WALTERS, W. (1955), Arch. Surg. (Chicago), 70, 323.

WAUGH, J. M., WALTERS, W., GRAY, H. K and PRIESTLEY,'J. T. (1952), Proc. Staff Meet. Mayo Clin., 27, 578. 